\title{
A new peroxisomal disorder with fetal and neonatal adrenal insufficiency
}

\author{
Christine Vanhole, Francis de Zegher, Paul Casaer, Hugo Devlieger, Ronald J A Wanders, \\ Geertrui Vanhove, Jaak Jaeken
}

\begin{abstract}
A boy with a new type of adrenoleukodystrophy is described. This was characterised by fetal and neonatal adrenal insufficiency, a neurological picture as seen in neonatal adrenoleukodystrophy, but with a normal number of peroxisomes in the liver and a peroxisomal dysfunction limited to the very long chain fatty acids and pristanic acid.
\end{abstract}

(Arch Dis Child 1994; 71: F55-F56)

The peroxisomal disorders are a group of inherited diseases due to an impairment of the peroxisomal assembly or of one or more peroxisomal functions. ${ }^{1}$ They have been classified into three categories. In the first group, peroxisomes are absent leading to a generalised peroxisomal dysfunction. One of them is neonatal adrenoleukodystrophy (NALD), an autosomal recessive lipid storage disease with profound hypotonia and intractable seizures. Zellweger (cerebrohepatorenal) syndrome and infantile Refsum's disease also belong to this group. In the second group, peroxisomes are present and several peroxisomal functions are impaired (for example, the rhizomelic form of chondrodysplasia punctata). In the third group, peroxisomes are present and only a single peroxisomal function is impaired (for example, defective transport of the activating enzyme of very long chain fatty acids (VLCFA) in X linked adrenoleukodystrophy (ALD).

Here we report on an infant with a peroxisomal disorder distinct from known peroxisomal disorders.

University of Leuven, Belgium, Department of Paediatrics

Christine Vanhole

Francis de Zegher

Paul Casaer

Hugo Devlieger

Jaak Jaeken

\section{Department of}

Pharmacology

Geertrui Vanhove

Department of

Paediatrics, University

of Amsterdam, The

Netherlands

Ronald J A Wanders

Correspondence to:

Dr J Jaeken, Department of Paediatrics, University

Hospital Gasthuisberg

Herestraat 49, 3000 Leuven, Belgium.

Accepted 9 March 1994

\section{Case report}

A boy was born after a 39 weeks' gestation as the second son of healthy, unrelated parents. The older sibling was 2 years old and healthy. Pregnancy was complicated by severe intrauterine growth retardation of unknown origin, oligohydramnios, and premature onset of labour. The mother reported normal fetal activity. Maternal serum concentrations of oestriol were measured seven times at weekly intervals (33-39 weeks) and were always undetectable, whereas the concentrations of human placental lactogen were repeatedly in the low-normal range.

Birth weight was $2120 \mathrm{~g}$ (10th centile $2500 \mathrm{~g}$ ), length $44.5 \mathrm{~cm}$ (10th centile 45.3 $\mathrm{cm})$, and head circumference $34 \mathrm{~cm}$ (50-75th centile). On the third postnatal day the infant was referred because of hypotonia and feeding difficulties. Upon admission the child was extremely hypotonic. Moro, grasp, and deep tendon reflexes were absent. He had a slightly dysmorphic face with fish mouth and high arched palate. There was bilateral cryptorchidism. He had a holosystolic cardiac murmur due to a small ventricular septal defect. Convulsions were first noted on day 10 and responded poorly to phenobarbitone, phenytoin, and diazepam.

Plasma and fibroblast concentrations of VLCFA were markedly raised; on day 9 plasma $\mathrm{C} 24 / \mathrm{C} 22$ was 2.53 (normal range $0.50-0.90$ ) and plasma $\mathrm{C} 26 / \mathrm{C} 22 \quad 0.55$ (normal range $0.01-0.03) .^{2}$ Plasma pristanic acid was also increased (8.95 $\mu \mathrm{mol} / 1$; normal range $0.01-3$ $\mu \mathrm{mol} / \mathrm{l}){ }^{1}$ The peroxisomal fatty acid $\beta$ oxidation enzyme activities in liver were normal ${ }^{3}$; immunoblot analysis of the peroxisomal $\beta$ oxidation enzyme proteins in cultured skin fibroblasts also gave normal results. ${ }^{4}$ A liver biopsy specimen revealed haemosiderosis and a normal number of peroxisomes. However, the peroxisomes were pleiomorphic and showed a dense inclusion ('nucleoid'; figure). Nucleoids have been reported in animals but as far we know not in humans.

Somatosensory evoked potentials showed a central conduction deficit. Peripheral nerve conduction velocity was initially normal and slowed down later on, suggesting a defective myelinisation. Computed tomography of the brain showed hypodensity of the white matter.

Normal results were obtained for liver function tests, chromosome analysis, screening for other metabolic disorders, electroencephalography, visual evoked potentials, brain stem auditory evoked responses, electromyography, and ophthalmological examination.

The patient showed striking episodes of acute cardiovascular deterioration, weakness,

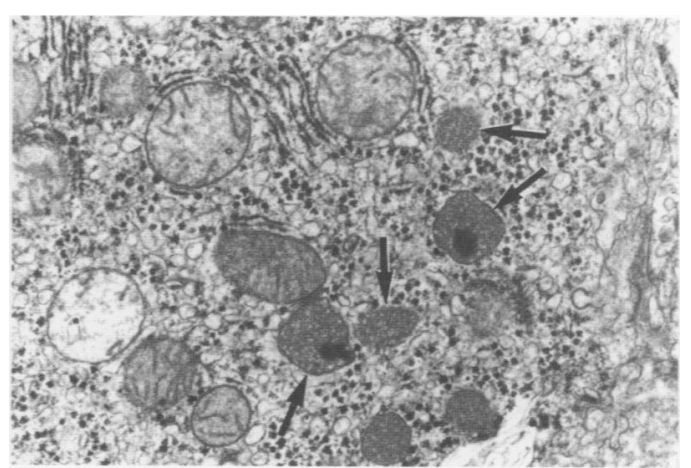

Electron photomicrograph of peroxisomes (arrows) in liver biopsy showing polymorphism and the presence of a nucleoid (magnification $\times 28750$ ). 
and increased frequency of convulsions. These insults became gradually more severe. Endocrine investigations suggested adrenal insufficiency. Indeed, during these episodes plasma sodium concentrations were low (around $132 \mathrm{mmol} / \mathrm{l}$ ) and there was an increased number of blood eosinophils $(12-17 \%)$. Plasma cortisol (33.1-173.8 $\mathrm{nmol} / \mathrm{l})$, dehydroepiandrosterone sulphate $(<130 \mathrm{nmol} / \mathrm{l})$ and aldosterone $(721 \cdot 2 \mathrm{pmol} / \mathrm{l})$ were low, while plasma adrenocorticotrophic hormone was increased $(56.6 \mathrm{pmol} / \mathrm{l})$. Plasma transcortin, $17 \alpha-\mathrm{OH}$-progesterone, testosterone, and renin were normal. Hypoglycaemia was not documented but the infant received continuous parenteral nutrition. After administration of hydrocortisone there was a significant though transient improvement of the general condition and a decrease in the frequency of the seizures. The boy died at the age of 6 weeks after milk aspiration. Necropsy was not performed.

\section{Discussion}

The present patient showed a clinical picture compatible with NALD. ${ }^{5}$ However, contrary to the near absence of liver peroxisomes in NALD liver peroxisomes were present and peroxisomal functions appeared intact except for markedly raised VLCFA and pristanic acid concentrations. The peroxisomal fatty acid $\beta$ oxidation, after activation, involves at least three enzymatic steps performed by acyl-CoA oxidase, bifunctional enzyme, and thiolase. In NALD an abnormality of peroxisome biogenesis results in a generalised peroxisomal dysfunction. Recently, several children have been described with a phenotype resembling Zellweger syndrome or NALD, but in whom peroxisome biogenesis was intact and a deficiency of only one of the enzymes involved in the peroxisomal fatty acid oxidation was found. ${ }^{1}$ In the present patient, no accumulation of bile acid intermediates was found. As a deficiency of either bifunctional protein or thiolase leads to the accumulation of VLCFA as well as bile acid intermediates one would expect a deficiency of palmitoyl-CoA oxidase. However, immunoblot analysis of palmitoylCoA oxidase in human liver and skin fibroblasts revealed the presence of this enzyme. Moreover, palmitoyl-CoA oxidase activity was normal, excluding a functional defect of the peroxisomal palmitoyl-CoA oxidase.

Another possible explanation for the isolated accumulation of VLCFA could be a deficiency of the peroxisomal very long chain acyl-CoA synthetase, as is the case in X linked ALD. The clinical picture in our patient, however, does not correspond to any of the phenotypes described for $\mathrm{X}$ linked ALD, and the plasma values of $C 24 / C 22$ and $C 26 / C 22$ in our patient are much higher than those described for $\mathrm{X}$ linked ALD. ${ }^{6}$

We do not seem to be able to explain our findings with the present peroxisomal $\beta$ oxidation model. A hypothetical explanation is the deficiency of a yet undescribed carrier or transport protein necessary for the oxidation of VLCFA, but not for the oxidation of 2-methyl branched fatty acids and bile acid intermediates. It also remains possible that further $\beta$ oxidation is not common to the oxidation of VLCFA and bile acid intermediates. Further research will be necessary to reveal the underlying defect in this patient.

Nevertheless, this report demonstrates again that an apparently selective defect of peroxisomal fatty acid $\beta$ oxidation may cause multiple organ pathology and severe neurological disturbances as observed in disorders of peroxisome biogenesis. Adrenal insufficiency is known to be associated with the $\mathrm{X}$ linked variant of childhood ALD. ${ }^{16}$ Necropsy has revealed adrenocortical atrophy in NALD. ${ }^{15}$ This infant represents the first case with a peroxisomal disorder in which fetal and neonatal adrenocortical insufficiency has been documented. As the serum testosterone concentration was normal, it is unlikely that the adrenocortical insufficiency was due to an enzymatic defect of steroid biosynthesis. At present, the mechanisms underlying the early and selective functional disintegration of the adrenal cortex are unclear.

We gratefully acknowledge Dr G Vrijens, obstetrician, for serial serum oestriol measurements in the mother, Dr Sc G Dacremont, department of paediatrics, University of Ghent, for plasma peroxisomal function analysis, Dr C Jacobs, department of paediatrics, Free University Hospital of Amsterdam, the Netherlands, for peroxisomal function analysis, Dr Sc R De Vos, department of pathology, University of Leuven, for electron microscopy of liver biopsy.

Geertrui Vanhove is aspirant of the Belgian Nationaal Fonds Geertrui Vanhove is aspirant of the
voor Wetenschappelijk Onderzoek'.

1 Wanders RJA, Schutgens RBH, Barth PG, van den Bosch H. Postnatal diagnosis of peroxisomal disorders: a biochemical approach. Biochimie 1993; 75: 269-79.

2 Wanders RJA, van Roermund CWT, van Wijland MJA, et al. Peroxisomal very long chain fatty acid beta-oxidation in cultured skin fibroblasts: activity in Zellweger syndrome and other peroxisomal disorders. Clin Chim Acta 1987; 166: 255-63.

3 Vanhove GF, Van Veldhoven PP, Fransen M, et al. The CoA esters of 2-methyl-branched chain fatty acids and the bile acid intermediates di- and trihydroxycoprostanic acids are oxidized by one single peroxisomal branched chain axyl-CoA oxidase in human liver and kidney. $\boldsymbol{f}$ Biol Chem acyl-CoA oxidase in hum

4 Tager JM, ten Harmsen van der Beek WA, Wanders RJA, et al. Peroxisomal beta-oxidation enzyme proteins in the Zellweger syndrome. Biochem Biophys Res Commun 1985; 126: 10269-75.

5 Kelley RI, Data NS, Dobyns WB, et al. Neonatal adrenoleukodystrophy: new cases, biochemical studies, and differentiation from Zellweger and related polydystrophy syndromes. Am 7 Med Genet 1986; 23: 869-901.

6 Moser HW, Bergin A, Naidu S, Ladenson PW. Adrenoleukodystrophy. Endocrinol Metab Clin North Am 1991; 20: 297-318. 